\title{
Cultural Differences in Mixed Emotions: The Role of Dialectical Thinking
}

\author{
Wen Zheng ${ }^{1,2 *}$, Ailin $\mathrm{Yu}^{2}$, Disi $\mathrm{Li}^{3}$, Ping Fang ${ }^{4}$ and Kaiping Peng ${ }^{2 *}$ \\ ${ }^{1}$ Department of Medical Psychology, Capital Medical University, Beijing, China, ${ }^{2}$ Department of Psychology, Tsinghua \\ University, Beijing, China, ${ }^{3}$ The Professional Qualification Authority of Ministry of Transport, Beijing, China, ${ }^{4}$ College of \\ Psychology, Capital Normal University, Beijing, China
}

OPEN ACCESS

Edited by:

Panagiotis Rentzelas, Birmingham City University, United Kingdom

Reviewed by:

William Tsai,

New York University, United States Li Jie, Inner Mongolia Normal University,

China

*Correspondence: Wen Zheng

xiaofeixia_z@163.com Kaiping Peng pengkp@mail.tsinghua.edu.cn

Specialty section: This article was submitted to Cultural Psychology, a section of the journal

Frontiers in Psychology

Received: 28 February 2020

Accepted: 18 November 2020

Published: 11 January 2021

Citation:

Zheng $W$, Yu A, Li D, Fang $P$ and Peng K (2021) Cultural Differences in

Mixed Emotions: The Role of Dialectical Thinking

Front. Psychol. 11:538793.

doi: 10.3389/fpsyg.2020.538793
Who can feel both happy and sad at the same time, but not discomfort? This study aimed to investigate the cultural differences in mixed emotional experiences induced by conflict stimuli among American and Chinese undergraduate students. In total, 160 Americans and 158 Chinese watched two different valence advertisements (one predominantly positive and the other predominantly negative) that elicited mixed emotions; their feelings were assessed through self-reported measures. Findings indicated the impact that cultural differences have in people's mixed emotional experiences depends on the emotional components of the mixed emotional situations. The Americans and Chinese both experience a comparably intense mixture of emotions in different valence situations, but their discomfort toward conflicting stimuli is different. Further, dialectical thinking may be a mechanism behind the influence of cultural differences in people's mixed emotional experiences. Implications for emotion theory and research are discussed.

Keywords: culture, dialectical thinking, mixed emotions, discomfort, thinking style

\section{INTRODUCTION}

On November 16, 2015, Oxford Dictionaries announced that their "Word of the Year" was an emoji: "Face with Tears of Joy." It was the first time that Oxford Dictionaries chose a pictograph as its "Word of the Year," representing a break with past winners, which were mostly old-fashioned, string-of-letters-type words. This emoji represents the experience of sadness mixed with joy, a "bittersweet" feeling. Mixed emotions represent a subset of emotion blends, which were defined as the co-occurrence of any two or more same-valence or opposite-valence emotions (Larsen and McGraw, 2011, 2014). This study surveyed 160 American and 157 Chinese undergraduate students as well as private sector employees to examine cultural differences in the experience of mixed emotions between the two groups. They were assessed through selfreported measures, using different valence advertisements as means to reflect peoples' complex emotional experiences in response to emotion inducing stimuli.

Previous research demonstrates that the experience of mixed emotions is influenced by response format (Schimmack et al., 2001, unpublished), one's openness (de Rivera, 1984; Tang and Singer, 1997), resilience (Ong and Bergeman, 2004), and age (Coats and Blanchard-Fields, 2008; Ersner-Hershfield et al., 2008). Moreover, mixed emotions have a positive impact on a person's well-being; hence, it may buffer the negative consequences of stressful events on an individuals' health (Berrios et al., 2018). Some researchers began to focus on the influence of 
culture on the experience of mixed emotions, suggesting that East Asians experience more mixed emotions than North Americans (Bagozzi et al., 1999; Kitayama et al., 2000). Thus, our study targets this research question. We aimed to investigate cultural differences in the experience of mixed emotions, as well as to explain how the dialectical thinking style may affect undergraduate students' experiences of mixed emotions.

Cross-cultural studies have repeatedly documented that the Western and the Eastern epistemologies and systems of thought diverge in important ways (Peng and Nisbett, 1999; Hamamura et al., 2008; Miyamoto et al., 2010). Eastern philosophies are largely rooted in Confucianism, Taoism, and Buddhism, which can be described as dialectical. Dialectical thinking features the three principles of contradiction (i.e., two opposing positions can both be true), change (i.e., two opposing positions may lie on different points of a temporal continuum), and holism (i.e., all things in the universe are interrelated). Western culture, which is largely based on Greek and Aristotelian logic, can be characterized as linear or synthesis-oriented. These logical thinking styles feature three key principles: the law of identity (i.e., if anything is true, then it is true), the law of excluded middle (i.e., any statement is either true or false), and the law of non-contradiction (i.e., no statement can be both true and false; Peng and Nisbett, 1999). This thinking tradition tends to limit the degree to which Westerners engage in dialectical processing.

A growing number of cross-cultural research studies indicate that East Asians engage in dialectical thinking, and that this propensity may also influence their emotions (Spencer-Rodgers et al., 2010). People in independent-based cultures (e.g., the United States) tend to experience emotions in opposite ways, whereas people in interdependent-based cultures (e.g., China) experience emotions in dialectic ways (Bagozzi et al., 1999). In summary, dialecticism sees opposite-valence emotions (e.g., mixed emotions) as compatible with each other, whereas Western philosophies consider these emotions to be conflicting.

Previous research mainly focused on two aspects of cultural differences in mixed emotions: the frequency and the intensity of the mixed emotional experience; Bagozzi et al. (1999) had Asian and American subjects recall and indicate the frequency with which they experienced positive and negative emotions and found that the frequency estimates of positive and negative emotions were positively correlated for Asians, whereas negative correlations were found for Americans. Kitayama et al. (2000) asked participants from Japan and America to estimate the frequency in which they experienced positive and negative emotions; they found similar correlations between positive and negative emotions: they were less negative in dialectic cultures than in non-dialectic cultures, and, based on the correlational indices of co-occurrence. This led to the conclusion that Asians experience mixed emotions more frequently than Westerners. However, although positive correlations between two variables may imply this, they co-occur frequently. For example, strong negative correlations do not necessarily imply that they co-occur less frequently (Zelenski and Larsen, 2000; Larsen et al., 2017). Additionally, there is a concern about what has been reported as "mixed emotions," as specific reports might reflect people's semantic knowledge about the stimulus they experience during scientific experiments (Russell, 2017; Itkes et al., 2019). Therefore, these aforementioned mixed emotion reports could have reflected people's semantic judgments instead of their actual mixed emotional feelings.

Some researchers focused on how thinking style affects the intensity of the mixed emotional experience. To investigate the mechanism behind the notion that dialectical thinkers experience greater mixed emotions than non-dialectical thinkers, SpencerRodgers et al. (2010) experimented with Chinese and EuroAmerican undergraduates by making them experience mixed emotion life events. The results showed that Chinese people tend to engage in dialectical thinking; they exhibit greater mixed emotional experience than Euro-Americans, and dialecticism mediated participants' mixed emotional experiences, reinforcing the significance of cultural differences. However, Leu et al. (2010) found no evidence that dialectical thinkers experience more mixed emotions than non-dialectical thinkers. In their study, participants reported their emotional response toward standardized positive, negative, and mixed situations that depicted episodes in a protagonist's daily life. Results showed that cultural differences in the opposing emotions associations were found in positive events, and not in mixed or negative ones.

We may infer from the inconsistency of the aforementioned studies that the overall valence of the situation may have a mediation effect on how cultural differences affect mixed emotional experiences. Indeed, Miyamoto et al. (2010) found that the Japanese reported more mixed emotions than the Americans in predominantly pleasant situations (PPS), whereas there were no cultural differences in mixed emotions in predominantly unpleasant situations (PUS) or the mixed situations. Corroborating Miyamoto's findings, Hui et al. (2009) had Chinese participants report their emotional experiences to a positive and a negative event every week over 15 weeks, and they found an interaction between dialectical thinking and event valence. Specifically, non-dialectical thinkers tended to experience more mixed emotions in positive than in negative events.

Additionally, traditional model on mixed emotional experience concentrated on the associated negative consequences (e.g., anxiety and stress; Williams and Aaker, 2002). According to the Cognitive Dissonance Theory (Festinger, 1957), the experience of conflict creates uncomfortable tensions or discomfort; Chinese, who are more prone to dialectical thinking, are more likely to accept contradiction in reality and to synthesize contradiction than Westerners, who are more prone to linear thinking (Peng and Nisbett, 1999). Thus, cultural differences in the attitude toward contradictions manifest themselves through emotional constructs, which are manifested based on the extent to which the contradictory elements elicit them; for example, Westerners exhibit tension or discomfort when presented with situations that elicit cognitive, emotional, or behavioral contradictions (Cacioppo and Berntson, 1994). In that topic, Oceja and Carrera (2009) investigated different processing patterns of mixed emotions and found that highly simultaneous pattern elicited greater tension than sequential and prevalent patterns. 
In order to account for the emotional nature of a given situation (Miyamoto et al., 2017), we induced mixed emotions by presenting two different advertisements with varying valences (Williams and Aaker, 2002). We hypothesized that (H1) both the Chinese and American people are capable of experiencing mixed emotions, but the intensity of the experience varies depending on the situation; specifically, in PPS, the Chinese experienced more mixed emotion than the Americans, whereas in PUS, the Americans experienced more mixed emotion than the Chinese. Notably, the Chinese are more inclined to engage in dialectical thinking, and they tend to deal with apparent contradictions in a compromising way, which may advocate tolerance of more blended emotions. Thus, we inferred that there is a difference between how the Americans and Chinese experience contradictions. Further, we have also tried to explain how the dialectical thinking style may affect the discomfort elicited by confronting opposing emotional stimuli. We hypothesized that $(\mathrm{H} 2)$ the Chinese will tend to engage in dialectical thinking, and in PPS, it will be easier for them to identify negative meanings in positive events; as such, they will experience more mixed emotions and discomfort. Contrastingly, Americans will tend to engage in linear thinking, and in PPS, they will try to amplify the significance of positive emotions, thus reducing the impact of negative emotions; in doing so, they will experience less intense mixed emotions regarding the same situation. However, in PUS, both the Chinese and Americans may experience the same level of mixed emotion and discomfort. Owing to this, the Chinese try to find positive meanings in negative events, whereas the Americans have to be motivated to regulate their negative emotions. Regarding mixed emotion calculation, early studies used variable correlations to index mixed emotional experience (Russell, 1980; Watson and Tellegen, 1985; Remmington et al., 2000). To address the limitations of using correlations, we applied two co-current indexes to indicate the intensity of mixed emotional experience.

\section{MATERIALS AND METHODS}

\section{Participants}

In total, 160 American individuals participated in this study. Among them, there were 57 undergraduates $(18$ males, 39 females, mean age $=20.32$ years) from the University of California, Berkeley, and 103 individuals (55 males, 48 females, mean age $=39.35$ years) were recruited from Amazon Mechanical Turk. All the American participants were Anglo-, African-, Hispanic-, or Indian-Americans, including 80.0\% AngloAmericans, $11.25 \%$ African-Americans, $6.25 \%$ HispanicAmericans, and 2.5\% Indian-Americans. In total, 158 Chinese individuals participated in this study. Among them, there were 122 undergraduate students ( 32 males, 90 females, mean age $=19.80$ years) from the Capital Normal University in Beijing, China, and 36 individuals $(20$ males, 16 females, mean age $=29.74$ years) were recruited from an advanced technological enterprise in Shanghai. Undergraduate participants were recruited from both universities' Introduction to Psychology for course credit. American participants from Amazon Mechanical Turk and Chinese workers were paid.

This study was carried out in accordance with the recommendations of the guidelines of Human Research Ethics Committees of both the Capital Normal University and the University of California, Berkeley. All participants provided written informed consent prior to their participation. We affirm that such consent was in accordance with the Declaration of Helsinki. The protocol was approved by the Human Research Ethics Committees of both the Capital Normal University and the University of California Berkeley.

\section{Materials}

\section{Advertisements}

Two advertisements were used in the study as conflict stimuli (Williams and Aaker, 2002). Each advertisement included a colorful photograph and a corresponding, contradictory advertising message (that reflected the opposite emotion of the image); this was intended to elicit two different emotional ambivalence states: predominantly pleasant and predominantly unpleasant mixed emotions, respectively.

Participants in the PPS read the message "My grandpa passed away years ago. He was a college professor and he dedicated his whole life to education. I loved spending time with him. I'm happy that he was alive long enough to get to know me and help raise me"; in the PUS, they read "I have been dreading this moment, but it has finally arrived. A chapter in my life is ending, and the future is still uncertain. I'll miss the neighborhood and the friends I've made. I really do not want to leave. It's a sad and nostalgic time." This message elicits a predominantly unpleasant mixed emotion with stronger feelings of sadness than happiness.

To enhance external validity, positive-valence product content was included in the advertisement to enhance external validity and was consistently presented in all conditions. For example, one positive-valence content stated, "Watson color film has top color quality-plus; the texture will always be sharp, never grainy. Just like life."

\section{Dialectical Self Scale}

The 32-item Dialectical Self Scale (DSS; Spencer-Rodgers et al., 2001, unpublished) was designed to assess subjects' ability to think dialectically. The scale is composed of three subscales: contradiction, cognition change, and behavior change. Here is a sample item: "When I hear two sides of an argument, I often agree with both." Participants rated each item on a 7-point Likert scale ranging from 1 (strongly disagree) to 7 (strongly agree). The DSS possesses adequate cross-cultural validity and reliability (Hamamura et al., 2008). In our study, the Cronbach's $\alpha$ values were 0.69 (Chinese) and 0.87 (Americans).

\section{Emotion Measurement}

Participants were asked to imagine themselves as in the situations described in the advertisements. Then, they rated nine different emotions on a 7-point scale from 1 (not at all) to 7 (very strongly), such as "If you were the grandson, how sad would 
you feel?" Positive emotions were happy, delighted, and joyful; negative emotions were sad, sorrowful, and depressed; discomfort emotions were tense and nervous. Positive affect (PA), negative affect (NA), and discomfort were separately computed by averaging each of their three corresponding items. A Factor analysis was conducted to explore whether discomfort items are different with the negative emotion items. Both discomfort and negative emotion items were loaded well (i.e., $0.727-0.829$ ) on a single factor $($ Eigen $=3.942,65.71 \%$ of the variance). But, the loading of tense, anxious, and nervous is higher than sad, sorrowful, and depression. In this study, the relative Cronbach's $\alpha$ values were $0.872(\mathrm{PA}), 0.759$ (NA), and 0.758 (discomfort).

We computed each participant's mixed emotional experience using Schimmack's (2001) MIN as one of the indexes of mixed emotions. Schimmack (2001) developed a direct measure of co-occurrence, indexing mixed emotions as the smaller one of a given observation's positive and negative emotion ratings [MIN (positive emotion, negative emotion)]. Higher MIN scores indicate greater mixed emotions, whereas lower scores indicate smaller mixed emotions. We also used the negative acceleration model (NAM; Scott, 1966) as another index of mixed emotions by applying the following formula: $([2 * S]+1) /(S+L+2)$, where $S$ is the smaller, and $L$ is the larger mean affect rating. Higher scores indicate greater mixed emotions (Spencer-Rodgers et al., 2010).

\section{Additional Measures}

We used one item to measure the extent to which participants experienced conflict, namely, "How conflicted would you feel in this type of event?" Participants rated the item on a 7-point scale from 1 (not at all) to 7 (very strongly).

All the experimental materials were written in English as well as in Chinese and applied to the corresponding native participants. Specifically, the Chinese version was applied after translating and back-translating with the assistance of doctoral candidates in Psychology, and to improve its adherence to Chinese cultural norms, this version underwent some minor adjustments when compared with the original English version.

\section{Procedures}

Participants were told that this study aimed to assess consumers' response to potential advertisements. They were instructed to view its appeal just as if they were reading it in a magazine. Randomly, participants were presented with three different advertisements. After reading each advertisement, participants were asked to rate their subjective feelings about the advertisement and respond to the other abovementioned items.

After finishing each emotional measurement, participants were instructed to copy a 3D sketch to avoid the overlapping of the induced emotions. Each emotional measurement took approximately $10 \mathrm{~min}$. After finishing one measurement, the students proceed to the next advertisement. The order of the presentation of the advertisements was counter-balanced.

Subsequently, participants were asked to complete the DSS and a demographic questionnaire. After the questionnaire, participants were debriefed and thanked for their participation.

\section{RESULTS}

\section{Manipulation Check}

The ratings of overall positive and negative emotions were analyzed to check for the general valence of each situation. The results of repeated measurement MANOVA revealed a significant main effect of situation on positive emotion, $F(1,315)=551.277$, $p<0.01, \eta^{2}=0.636$, and on negative emotion, $F(1,315)=250.207$, $p<0.01, \eta^{2}=0.584$. Least significance difference analyses indicated that participants in the PPS $(M=4.781 \pm 0.084)$ experienced significantly more positive emotions than those in the PUS $(M=2.469 \pm 0.077), p<0.001$; participants in the PUS $(M=3.867 \pm 0.091)$ experienced significantly more negative emotion than those in the PPS $(M=1.738 \pm 0.059), p<0.001$. No cultural differences were observed.

For each type of situation, we computed the number of participants that rated conflict greater than 0 (not at all), regardless of the intensity of the emotions. In the PPS, $53.1 \%$ of the participants reported perceived contradictions, whereas the rate was $90.3 \%$ in the PUS.

\section{Cultural Differences in Dialectical Thinking}

The independent sample $t$-test results showed significant differences between the Chinese and Americans, $t(315)=11.422$, $p<0.01$. Specifically, the dialectical thinking of Chinese participants $(M=4.275, S D=0.239)$ was significantly higher than that of American participants $(M=3.946, S D=0.273)$.

One-way ANOVA was conducted to compare the difference between different races on dialectical thinking. The results indicated that there were significant differences between the races, $F(4,317)=33.547, p<0.01$. Specifically, dialectical thinking in the Chinese was significantly more prevalent than that in the Anglo- $(M=3.932, S D=0.258)$, African- $(M=3.971$, $S D=0.370)$, and Hispanic-Americans $(M=4.039, S D=0.306)$; no significant differences were found between the Chinese and Indian-Americans $(M=4.072, S D=0.149)$.

\section{Cultural Differences in Mixed Emotions}

The results of repeated measurement ANOVA showed no significant effects of situation and culture or interaction between them on MIN scores, $p>0.05$, after controlling for sex, age (Larsen et al., 2007), and multicultural experience (Ritter et al., 2012). The descriptive statistics of the main variables are shown in Table $\mathbf{1}$.

A repeated MANOVA indicated no significant main effects of situation and culture or interaction between them on NAM scores, $p>0.05$, after controlling for sex, age, and multicultural experience.

\section{Cultural Differences in Discomfort Induced by Conflict Stimuli}

A repeated measurement ANOVA on discomfort was performed. Figure 1 presents the cultural differences in participants' discomfort induced by conflict stimuli in two different situations.

After controlling for sex, age, and background, a significant main effect of culture was found, $F(1,313)=128.707, p<0.001$, $\eta^{2}=0.291$; a main effect of situation, $F(1,313)=8.780, p<0.01$, 


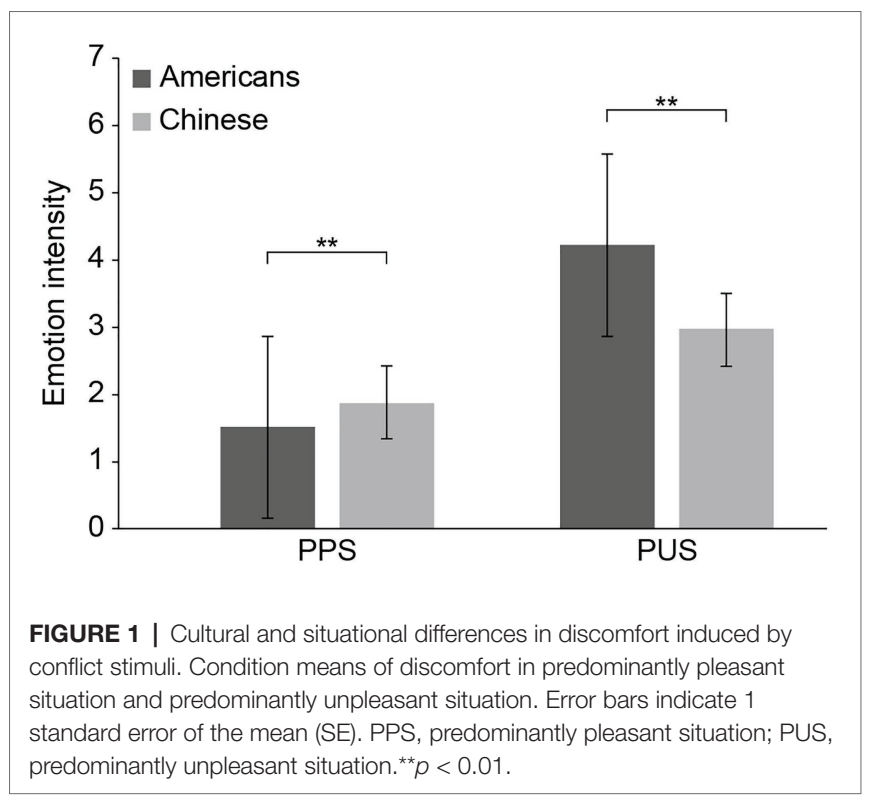

$\eta^{2}=0.027$, and culture and situation had significant interactions with discomfort, $F(1,313)=63.996, p<0.001, \eta^{2}=0.170$.

Results of simple effect analysis indicated that: in the PPS, Chinese participants significantly felt more discomfort than Americans, $t(315)=3.244, p<0.01, d=0.364$; in the PUS, American participants significantly felt more discomfort than Chinese, $t(315)=-7.087, p<0.001, d=0.796$.

\section{Mediation of Dialectical Thinking in the Relationship Between Culture and Mixed Emotional Experience}

The correlation of all variables mentioned before is shown in Table 2. We conducted mediation analyses on the two different situations separately (PPS and PUS).

In the PPS, results indicated that culture significantly negatively predicted dialectical thinking, $\beta=-0.543, p<0.001$. When culture and dialectical thinking both entered the model, dialectical thinking significantly positively predicted MIN, $\beta=0.188$, $p<0.05$, and discomfort, $\beta=0.213, p<0.01$. The direct effect of culture was not significant on MIN, NAM, and discomfort, $\beta_{\mathrm{MIN}}=-0.01, \beta_{\mathrm{NAM}}=-0.04, \beta_{\mathrm{DIS}}=-0.09, p>0.05$. The Bootstrap confidence intervals of the indirect effect of culture on MIN were $[-0.121,-0.009]$, indicating that dialectical thinking played a significant indirect effect on the relationship between culture and MIN, $\beta=-0.079, p<0.001$. The Bootstrap confidence intervals of the indirect effect of culture on NAM were $[-0.117,-0.001]$, indicating that culture had a significant indirect effect on NAM, $\beta=-0.061, p<0.001$. The Bootstrap confidence intervals of the indirect effect of culture on discomfort were $[-0.173,-0.059]$, indicating that culture had a significant indirect effect on discomfort, $\beta=-0.116, p<0.001$. Figure 2 shows the standardized values of our mediation model, and the model fit indexes are shown in Table 3.

However, in the PUS when culture and dialectical thinking both entered the model, the effects of dialectical thinking on 
TABLE 2 | Correlations between dialectical thinking and emotional experience induced by conflict stimuli $(N=318)$.

\begin{tabular}{|c|c|c|c|c|c|c|c|c|c|c|c|c|}
\hline & 1 & 2 & 3 & 4 & 5 & 6 & 7 & 8 & 9 & 10 & 11 & 12 \\
\hline 1. Culture & 1 & & & & & & & & & & & \\
\hline $\begin{array}{l}\text { 2. Dialectical } \\
\text { thinking }\end{array}$ & $-0.541^{* *}$ & 1 & & & & & & & & & & \\
\hline 3. PA1 & 0.018 & 0.027 & 1 & & & & & & & & & \\
\hline 4. NA1 & -0.007 & 0.087 & $-0.208^{* *}$ & 1 & & & & & & & & \\
\hline 5. MIN1 & -0.072 & $0.118^{*}$ & -0.044 & $0.876^{* *}$ & 1 & & & & & & & \\
\hline 6. NAM1 & -0.086 & 0.108 & -0.543 & $0.726^{* *}$ & $0.825^{* *}$ & 1 & & & & & & \\
\hline 7. Discomfort1 & $-0.180^{* * *}$ & $0.213^{* *}$ & 0.010 & $0.613^{* *}$ & $0.676^{* *}$ & $0.516^{* * *}$ & 1 & & & & & \\
\hline 8. PA2 & 0.026 & 0.053 & $0.241^{* *}$ & 0.068 & $0.115^{*}$ & -0.030 & $0.188^{* *}$ & 1 & & & & \\
\hline 9. NA2 & $0.122^{*}$ & -0.018 & $0.214^{* *}$ & $0.214^{* * *}$ & $0.202^{* *}$ & 0.038 & $0.130^{*}$ & $-0.288^{* *}$ & 1 & & & \\
\hline 10. MIN2 & 0.017 & 0.026 & $0.244^{* *}$ & $0.237^{* *}$ & $0.287^{* *}$ & 0.097 & $0.348^{* *}$ & $0.646^{* *}$ & $0.157^{* *}$ & 1 & & \\
\hline 11. NAM2 & -0.074 & 0.013 & 0.035 & $0.114^{*}$ & $0.159^{* *}$ & 0.109 & $0.248^{* * *}$ & $0.420^{* * *}$ & $-0.355^{* *}$ & $0.773^{* *}$ & 1 & \\
\hline 12. Discomfort2 & $0.371^{* *}$ & $-0.126^{*}$ & $0.150^{* *}$ & $0.185^{* *}$ & $0.195^{* *}$ & 0.087 & $0.158^{* *}$ & -0.046 & $0.647^{* *}$ & $0.210^{* *}$ & $-0.152^{* *}$ & 1 \\
\hline
\end{tabular}

PA1 represents the positive emotions in a predominantly pleasant situation. NA1 represents the negative emotions in a predominantly pleasant situation. MIN1 and NAM1 represent the mixed emotional experience in a predominantly pleasant situation. Discomfort1 represents the tense and nervous induced in a predominantly pleasant situation. PA2 represents the

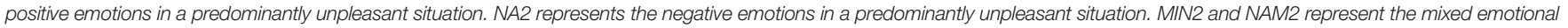
experience in a predominantly unpleasant situation. Discomfort2 represents the tense and nervous induced in predominantly unpleasant situation. ${ }^{*} p<0.05 ;{ }^{* *} p<0.01 ;{ }^{* * *} p<0.001$.

MIN, NAM, or discomfort were not significant, $\beta=-0.031$, $p>0.05$. Similarly, the direct effects of culture on MIN, NAM, or discomfort were not significant.

\section{DISCUSSION}

The present study investigated the cultural differences in people's mixed emotional experiences, including its byproducts (discomfort induced by conflict stimuli in different situations), and explain how differing thinking styles affect these experiences. The initial findings show no cultural differences in mixed emotions between the two groups, but cultural differences in discomfort. Dialectical thinking mediated these cultural differences in mixed emotional experience and discomfort. These results support our hypotheses. The present study expands on previous studies by exploring the role of dialectical thinking in the cultural differences in mixed emotion and its byproduct (discomfort).

\section{Cultural Differences in Mixed Emotional Experiences in Different Situations}

We analyzed the interaction between culture and advertisement messaging on different indexes of mixed emotions, MIN and NAM, as well as discomfort. No significant cultural differences were found in either MIN or NAM. Thus, both indexes of mixed emotions provided consistent evidence that Americans and Chinese experienced the same mixed emotions in the PPS and the PUS, and that Chinese felt more mixed emotions than Americans only in the PUS. The findings indicated that both Americans and Chinese have the ability to perceive and experience the mixed emotional stimuli; hence, when confronted with conflict, they both experience conflicting emotions. These findings support our hypothesis (H1) and coincide with those of Leu et al. (2010) and provide a valuable supplement to Spencer-Rodgers et al. (2010) work. Further, we also compared the cultural differences in different indexes of mixed emotions and found consistent evidence (Spencer-Rodgers et al., 2010).

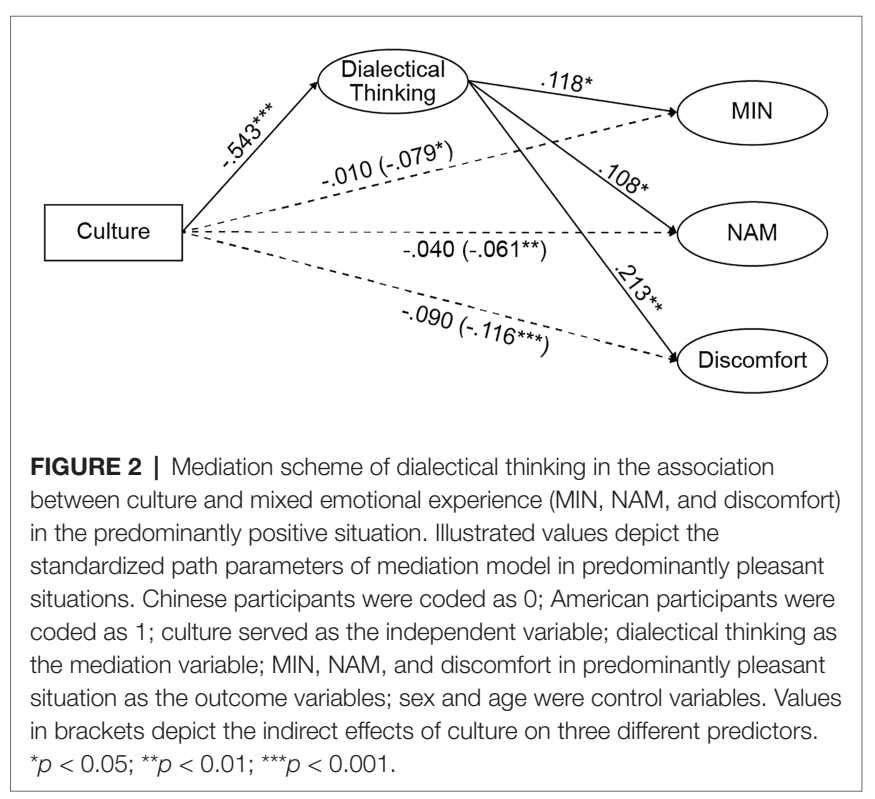

TABLE 3 | Model fit indexes of the mediation analysis in the predominantly positive situation.

\begin{tabular}{cccccccc}
\hline & CMIN/DF & NFI & RFI & IFI & TLI & CFI & RMSEA \\
\hline Model & 1.34 & 0.994 & 0.981 & 0.999 & 0.995 & 0.998 & 0.033 \\
\hline
\end{tabular}

However, results also showed that the same stimuli made Chinese and American participants experience different degrees of discomfort. First, the discomfort rose as the intensity of mixed emotions increased in both situations; however, the discomfort increased more in the PUS than in the PPS. Second, in the PPS, Chinese reported significantly more discomfort than Americans; in the PUS, Americans reported significantly more discomfort than Chinese.

This pattern of results has several important implications. First, the findings show that both the American and Chinese participants experienced comparable mixed emotions in response to positive and negative situations. Previous research on culture 
and mixed emotions has tended to neglect situational factors; we hope our situational account may explain some previous inconsistent findings on culture and mixed emotions (Hui et al., 2009; Miyamoto et al., 2010). Second, we explored the association between culture and discomfort that accompanies mixed emotions. Though previous studies claim that mixed emotions can make one tense and nervous, little attention has been paid to the cultural differences of discomfort when individuals were confronted with conflicting stimuli. To our knowledge, this study is the first attempt to explore the cultural differences in discomfort that accompany mixed emotions to deepen extant understandings of the experience of mixed emotions.

\section{Mediation of Dialectical Thinking in Cultural Differences in Mixed Emotional Experiences}

Why do American and Chinese people experience comparable mixed emotions, but different levels of discomfort in both positive and negative situations? Peng and Nisbett (1999) have suggested that a dialectical style of thinking may explain the cultural differences in contradiction processing. One plausible alternative mechanism is that because the Chinese tend to engage in dialecticism they therefore habitually process both positive and negative information with more ease.

The results of mediation analyses indicated that dialectical thinking does indeed play a complete mediating role in the impact of cultural differences in MIN, NAM, as well as in discomfort in the PPS. We believe that the PPS findings may be related to features of dialecticism, namely, the expectation for change and tolerance of contradictions (Peng and Nisbett, 1999). Dialecticism also dictates that the valence of an event is likely to change with time and perspective (Ji et al., 2001). This means that, when confronted with positive events, the Chinese tend to think that such happiness might endure for a short time, so they start looking for potential negative implications, that is, they start planning ahead for a future rainy day. Contrastingly, Americans tend to maximize the happy moment they are living because they tend to think they are currently experiencing it owing to a deeply rooted principle in the American culture: "Happiness is a basic right that everyone can pursue"; this principle detracts from paying too much attention to the fact that such positivity will not last forever.

Nevertheless, in the PUS, the mediating effect of dialectical thinking on cultural differences in mixed emotional experience was not observed. This finding denotes that dialectical thinking can interpret the cultural differences in mixed emotional experience in PPS, which is in accordance with the previous research that dialectical thinkers and non-dialectical thinkers differ in their level of mixed emotions only in positive events, but not in negative events (Hui et al., 2009). This may due to different mechanisms. Dialectical thinkers may be driven by their balanced event appraisal style to experience mixed emotions, whereas non-dialectical thinkers may be motivated by their self-affirmation to generate positive implication to cope with negative events (Larsen et al., 2003).

These results shed light on a thinking style account for why some individuals experience mixed emotions more discomfort.
Previous studies explore the association of dialecticism and mixed emotional experience, which is correlational, and cannot draw causal inferences (Hui et al., 2009). In this study, we introduced the mixed emotional experience and provided empirical support for the associations. This study addressed how an ideological thinking style can explain the cultural differences in mixed emotions and provided us a further insight into how people process and experience the ambivalence in different cultures. When studying mixed emotions in a global context, the cultural factors and the thinking style factors should be taken into account.

\section{CONCLUSION}

This study suggests that the impact that cultural differences have on people's mixed emotional experiences depends on the emotional components of mixed emotional situations. Further, it suggests that the American and Chinese both experience mixture of emotions in different valence situations, but their discomfort toward the conflicting stimuli is different. Additional dialectical thinking may be a mechanism behind the influence of cultural differences in people's mixed emotional experiences. Together, the findings contribute toward future theorizing on social cognition and emotion.

\section{DATA AVAILABILITY STATEMENT}

The datasets generated for this study can be found in the https://mfr.de-1.osf.io/render?url=https://osf.io/zrt3k/?direct\%26 mode $=$ render\%26action $=$ download $\% 26$ mode $=$ render .

\section{ETHICS STATEMENT}

The studies involving human participants were reviewed and approved by Human Research Ethics Committees of both Capital Normal University and University of California Berkeley. The patients/participants provided their written informed consent to participate in this study.

\section{AUTHOR CONTRIBUTIONS}

WZ, AY, and DL contributed equally to this work. WZ and KP served as the corresponding authors for the manuscript. PF and $\mathrm{KP}$ contributed significantly to the theory development, with KP contributing to research resources. DL contributed to the initial research design and data collection. WZ contributed to additional data collection, data analysis, as well as drafting and revising of the manuscript. AY contributed to the additional data collection, as well as the drafting and revising of the manuscript. All authors contributed to the article and approved the submitted version.

\section{ACKNOWLEDGMENTS}

We would like to thank Editage (www.editage.com) for the English language editing and publication support service. 


\section{REFERENCES}

Bagozzi, R. P., Wong, N., and Yi, Y. (1999). The role of culture and gender in the relationship between positive and negative affect. Cognit. Emot. 13, 641-672. doi: 10.1080/026999399379023

Berrios, R., Totterdell, P., and Kellett, S. (2018). When feeling mixed can be meaningful: the relation between mixed emotions and Eudaimonic wellbeing. J. Happiness Stud. 19, 841-861. doi: 10.1007/s10902-017-9849-y

Cacioppo, J. T., and Berntson, G. G. (1994). Relationship between attitudes and evaluative space - a critical-review, with emphasis on the separability of positive and negative substrates. Psychol. Bull. 115, 401-423. doi: 10.1037/0033-2909.115.3.401

Coats, A. H., and Blanchard-Fields, F. (2008). Emotion regulation in interpersonal problems: the role of cognitive-emotional complexity, emotion regulation goals, and expressivity. Psychol. Aging 23, 39-51. doi: 10.1037/0882-7974.23.1.39

de Rivera, J. (1984). The structure of emotional relationships. Rev. Pers. Soc. Psychol. 5, 116-145.

Ersner-Hershfield, H., Mikels, J. A., Sullivan, S. J., and Carstensen, L. L. (2008). Poignancy: mixed emotional experience in the face of meaningful endings. J. Pers. Soc. Psychol. 94, 158-167. doi: 10.1037/0022-3514.94.1.158

Festinger, L. (1957). A theory of cognitive dissonance. Redwood. CA: Stanford University Press.

Hamamura, T., Heine, S. J., and Paulhus, D. L. (2008). Cultural differences in response styles: the role of dialectical thinking. Pers. Individ. Differ. 44, 932-942. doi: 10.1016/j.paid.2007.10.034

Hui, C. M., Fok, H. K., and Bond, M. H. (2009). Who feels more ambivalence? Linking dialectical thinking to mixed emotions. Pers. Individ. Differ. 46, 493-498. doi: 10.1016/j.paid.2008.11.022

Itkes, O., Eviatar, Z., and Kron, A. (2019). Semantic and affective manifestations of ambi (valence). Cognit. Emot. 33, 1356-1369. doi: 10.1080/02699931. 2018.1564249

Ji, L. J., Nisbett, R. E., and Su, Y. (2001). Culture, change, and prediction. Psychol. Sci. 12, 450-456. doi: 10.1111/1467-9280.00384

Kitayama, S., Markus, H. R., and Kurokawa, M. (2000). Culture, emotion, and well-being: good feelings in Japan and the United States. Cognit. Emot. 14, 93-124. doi: 10.1080/026999300379003

Larsen, J. T., Hemenover, S. H., Norris, C. J., and Cacioppo, J. T. (2003). "Turning adversity to advantage: on the virtues of the coactivation of positive and negative emotions" in A psychology of human strengths: Perspectives on an emerging field. eds. L. G. Aspinwall and U. M. Staudinger (Washington, DC: American Psychological Association), 211-216.

Larsen, J. T., Hershfield, H. E., Stastny, B. J., and Hester, N. (2017). On the relationship between positive and negative affect: their correlation and their co-occurrence. Emotion 17, 323-336. doi: 10.1037/emo0000231

Larsen, J. T., and Mcgraw, A. P. (2011). Further evidence for mixed emotions. J. Pers. Soc. Psychol. 100, 1095-1110. doi: 10.1037/a0021846

Larsen, J. T., and Mcgraw, A. P. (2014). The case for mixed emotions. Soc. Personal. Psychol. Compass 8, 263-274. doi: 10.1111/spc3.12108

Larsen, J. T., To, Y. M., and Fireman, G. (2007). Children's understanding and experience of mixed emotions. Psychol. Sci. 18, 186-191. doi: 10.1111/ j.1467-9280.2007.01870.x

Leu, J., Mesquita, B., Ellsworth, P. C., ZhiYong, Z., Huijuan, Y., Buchtel, E., et al. (2010). Situational differences in dialectical emotions: boundary conditions in a cultural comparison of North Americans and East Asians. Cognit. Emot. 24, 419-435. doi: 10.1080/02699930802650911

Miyamoto, Y., Ma, X., and Wilken, B. (2017). Cultural variation in pro-positive versus balanced systems of emotions. Curr. Opin. Behav. Sci. 15, 27-32. doi: 10.1016/j.cobeha.2017.05.014

Miyamoto, Y., Uchida, Y., and Ellsworth, P. C. (2010). Culture and mixed emotions: co-occurrence of positive and negative emotions in Japan and the United States. Emotion 10, 404-415. doi: 10.1037/a0018430

Oceja, L., and Carrera, P. (2009). Beyond a single pattern of mixed emotional experience. Eur. J. Psychol. Assess. 25, 58-67. doi: 10.1027/1015-5759.25.1.58

Ong, A. D., and Bergeman, C. S. (2004). The complexity of emotions in later life. J. Gerontol. 59, P117-P122. doi: 10.1093/geronb/59.3.P117

Peng, K., and Nisbett, R. E. (1999). Culture, dialectics, and reasoning about contradiction. Am. Psychol. 54, 741-754. doi: 10.1037/0003-066X.54.9.741

Remmington, N. A., Fabrigar, L. R., and Visser, P. S. (2000). Reexamining the circumplex model of affect. J. Pers. Soc. Psychol. 79, 286-300. doi: 10.1037/0022-3514.79.2.286

Ritter, S. M., Damian, R. I., Simonton, D. K., van Baaren, R. B., Strick, M., Derks, J., et al. (2012). Diversifying experiences enhance cognitive flexibility. J. Exp. Soc. Psychol. 48, 961-964. doi: 10.1016/j.jesp.2012.02.009

Russell, J. A. (1980). A circumplex model of affect. J. Pers. Soc. Psychol. 39, 1161-1178. doi: 10.1037/h0077714

Russell, J. A. (2017). Mixed emotions viewed from the psychological constructionist perspective. Emot. Rev. 9, 111-117. doi: 10.1177/1754073916639658

Schimmack, U. (2001). Pleasure, displeasure, and mixed feelings: are semantic opposites mutually exclusive? Cognit. Emot. 15, 81-97. doi: 10.1080/ 02699930126097

Scott, W. A. (1966). Brief report: measures of cognitive structure. Multivar. Behav. Res. 1, 391-395. doi: 10.1207/s15327906mbr0103_9

Spencer-Rodgers, J., Peng, K., and Wang, L. (2010). Dialecticism and the cooccurrence of positive and negative emotions across cultures. J. Cross-Cult. Psychol. 41, 109-115. doi: 10.1177/0022022109349508

Tang, Z., and Singer, J. L. (1997). Daydreaming styles, emotionality and the big five personality dimensions. Imag. Cogn. Person. 16, 399-414. doi: 10.2190/ ATEH-96EV-EXYX-2ADB

Watson, D., and Tellegen, A. (1985). Toward a consensual structure of mood. Psychol. Bull. 98, 219-235. doi: 10.1037/0033-2909.98.2.219

Williams, P., and Aaker, J. L. (2002). Can mixed emotions peacefully coexist? J. Consum. Res. 28, 636-649. doi: 10.1086/338206

Zelenski, J. M., and Larsen, R. J. (2000). The distribution of basic emotions in everyday life: a state and trait perspective from experience sampling data. J. Res. Pers. 34, 178-197. doi: 10.1006/jrpe.1999.2275

Conflict of Interest: The authors declare that the research was conducted in the absence of any commercial or financial relationships that could be construed as a potential conflict of interest.

Copyright (C) 2021 Zheng, Yu, Li, Fang and Peng. This is an open-access article distributed under the terms of the Creative Commons Attribution License (CC BY). The use, distribution or reproduction in other forums is permitted, provided the original author(s) and the copyright owner(s) are credited and that the original publication in this journal is cited, in accordance with accepted academic practice. No use, distribution or reproduction is permitted which does not comply with these terms. 